\title{
Pobreza en México: Factor de vulnerabilidad para enfrentar los efectos del cambio climático
}

\author{
Poverty in Mexico: Vulnerability factor for face the effects of climate \\ change
}

\author{
Mérida Martínez, Y.; Acuña Gamboa, L. A.; Editor Academico Prof. \\ Dr. Carlos A. Zuniga-Gonzalez
}

\author{
Y. Mérida Martínez \\ ylianader@gmail.com \\ Universidad Autónoma de Chiapas, Mexico \\ L. A. Acuña Gamboa \\ Universidad Autónoma de Chiapas, Mexico \\ Editor Academico Prof. Dr. Carlos A. Zuniga- \\ Gonzalez \\ Universidad Nacional Autónoma de Nicaragua, León. \\ Nicaragua, Nicaragua
}

\author{
Revista Iberoamericana de Bioeconomía y Cambio \\ Climático \\ Universidad Nacional Autónoma de Nicaragua, León, Nicaragua \\ ISSN-e: 2410-7980 \\ Periodicidad: Semestral \\ vol. 1, núm. 2, 2015 \\ czuniga@ct.unanleon.edu.ni
}

Recepción: 06 Junio 2015

Aprobación: 01 Octubre 2015

URL: http://portal.amelica.org/ameli/journal/394/3941749002/

DOI: https://doi.org/10.5377/ribcc.v1i2.2475

Autor de correspondencia: ylianader@gmail.com
Resumen: Los niveles de pobreza que se presentan en México constituyen una problemática que se agudiza cada vez más en las diferentes escalas territoriales. La escasez de recursos económicos para el acceso a bienes, servicios y el factor cultural, constituyen la principal restricción para acceder a un nivel de vida adecuado, el cual proporcione condiciones integrales de seguridad en la población. Dicha condición resulta un factor determinante en la imposibilidad para hacer frente a los distintos fenómenos hidrometeorológicos y geológicos que se presentan como parte de las alteraciones climáticas en el sistema terrestre, situación que vulnera a la población en condición de pobreza a padecer los estragos en todas sus dimensiones: física, económica y social. El cambio climático, visto como un proceso que ha venido agudizándose debido a la actividad antrópica en las diferentes escalas territoriales, debilita la capacidad de respuesta y prevención de desastres ocasionados por éste, no sólo en los grupos vulnerables, más bien, en la intervención de las instancias gubernamentales, lo que conlleva a momentos de inestabilidad política, económica y social en los diferentes niveles de actuación (local, estatal y nacional).

Palabras clave: Pobreza, vulnerabilidad, variación climática, política pública, adaptación.

Abstract: Poverty levels that occur in Mexico are a problem that is becoming increasingly acute in the different territorial levels. The shortage of financial resources for access to goods, services and cultural factor, constitute the main constraint to access an adequate standard of living, which provide comprehensive security conditions in the population. This condition is a determining factor in the inability to deal with the various hydrometeorological and geological phenomena that occur as part of climatic changes in the Earth system, a situation that undermines the population living in poverty to suffer the ravages in all dimensions: physical, economic and social. Climate change, seen as a process that has been more acute due to human activity in the different territorial levels weakens the ability of response and prevention of disasters caused by it, not just vulnerable groups, rather, intervention government agencies, leading to moments of political, economic and social instability in the different action levels (local, state and national). 
Keywords: Poverty, vulnerability, climatic variation, public policy, adaptation.

\section{INTRODUCCIÓN}

"El cambio climático puede afectar negativamente a los objetivos de reducción de pobreza de Mexico". Banco Mundial

Hablar de pobreza obliga exponer una problemática social muy compleja que abarca múltiples dimensiones y que superan lo material y los ingresos; por ello han surgido diversos enfoques que ayudan al análisis de ésta desde diferentes visiones, sobre los cuales se hace, además, su medición, por ejemplo:

a) La carencia, escasez y la privación que hacen referencia a un conjunto de bienes y servicios materiales básicos, que permiten satisfacer las necesidades de las personas.

b) La exclusión social, vista también como un problema multidimensional que considera carencias de ingreso, vivienda, empleo, salud, educación y seguridad, ligado al rol que juegan las instituciones en este proceso social.

c) Las capacidades, como carencia y privación de ellas, incorpora las causas y las implicaciones de la política pública (Di Virgilio, Otero \& Boniolo, 2011).

d) Los ingresos mínimos, a los que un individuo tiene acceso para satisfacer sus necesidades, bienes y servicios. Considera una línea de pobreza que actualmente es de un dólar y medio al día, por lo que quienes se encuentran debajo de ésta son pobres, de acuerdo a la reducida o nula acumulación de capital económico (Boltvinik y Damián, 2013).

La situación de pobreza que se vive actualmente en las diferentes regiones del mundo representa un desafío que debe ser tomado en cuenta por los hacedores de políticas públicas y otras instancias. La presión que existe por los efectos del cambio climático es condición que contribuye, aún más, al empobrecimiento de las regiones y la dificultad para emerger de tal condición social, debido a las implicaciones que conlleva el modo de producción a la globalización.

En este sentido, la globalización entendida como la relación simbiótica de los espacios globales y locales (Bauman, 2013), abre la zona idónea de creación de necesidades superfluas en la sociedad. En la reestructuración del espacio a las tendencias globales, la sociedad coexiste y cohabita en una zona de consumo desmedido propia del mundo capitalista en el que vivimos. Inmersos en este sistema, la zona de consumo “[...] genera ávidos consumidores que se insertan dentro de este tipo de sociedad. Nuestra sociedad es consumista en el sentido que el poder hegemo\#nico esta\# centrado alrededor de empresas transnacionales que desean vender productos en escala masiva." (Acuña Gamboa, 2013:11-12).

En esta relación entre el tener funcional y el tener consumista (Fromm, 2012), las grandes empresas trabajan al ritmo de la demanda de una sociedad en constante búsqueda de lo novedoso, del bluf "necesario" para alcanzar el, por de más impuesto, paradigma del buen estilo de vida. Esta situación de producción en masa y de vida consumista ha generado un aumento de la actividad industrial, inherente a la generación de gases de efecto invernadero y la acumulación de desechos sólidos que son factores detonantes de la variación climática que actualmente afecta diferentes regiones del mundo.

Es un hecho que los países generadores de mayor cantidad de Gases de Efecto Invernadero (GEI) no han cumplido con los compromisos para reducirlos; por el contrario, las emisiones actuales han superado las del inicio de siglo. Por lo anterior, el Panel Intergubernamental para el Cambio Climático de las Naciones

Notas DE AUTOR 
Unidas (IPCC, por sus siglas en inglés) denuncia este hecho y agrega que es posible frenar este tipo de acciones haciendo uso adecuado de la tecnología (Restrepo, 2014). Estos GEI emitidos por las potencias mundiales, están repercutiendo a escalas globales y locales, haciendo que los países no industrializados sufran los efectos adversos de esta contaminación y; por ende, del deterioro ambiental.

A medida que el impacto del cambio climático se hace presente, las naciones han buscado estrategias para reducir sus efectos, al mismo tiempo que se crean estrategias de asistencia técnica y financiera lideradas por el Banco Mundial en los países en desarrollo, como parte de los compromisos internacionales para mitigar los efectos del cambio climático (BM, 2014), pues no debe olvidarse que habitamos en un sistema terrestre, cuyas repercusiones locales afectan a escala global, alterando los engranes de dicho sistema a velocidades impensables; por ello, algunos organismos internacionales (PNUD, IPCC y BM, por citar ejemplos) "[reconocen] la importancia de la reducción del riesgo[...] y hace llamados por estrategias de gestión[...]y de reducción de riesgo, incluyendo mecanismos como seguros, estrategias de la reducción del desastres y medios para manejar pérdidas y daños" (Quintero, Carvajal y Aldunce, 2012: 262).

Las desviaciones en las condiciones climáticas y las experiencias históricas constituyen un claro ejemplo de la presión que ejerce este cambio sobre la población pobre si no se toman las medidas adecuadas de adaptación, de lo contrario, estos sectores tendrían que tomar gran parte de sus ingresos para hacer frente a los efectos climáticos, situación que acrecienta la brecha de desigualdad y potencializa el riesgo en dicha población (OCDE, PNUD y BM, et.al., s/f). Por ende, al distribuir inadecuadamente las riquezas, esta brecha se hace cada vez más grande en un contexto determinado, cuando no se debiera olvidar que "[...]la reducción de la pobreza es un componente esencial para [minimizar] la vulnerabilidad frente a amenazas naturales[...]" (Quintero, Carvajal y Aldunce, 2012: 264).

Estos cambios drásticos en el clima tienen graves efectos sobre la sociedad pobre, característica de los países en desarrollo. Los efectos del cambio climático-como sequías y lluvias torrenciales-implican pérdidas materiales y humanas para la población en general; sin embargo, este tipo de eventos afectan a las personas que más carecen de ingresos y seguridad social (BM, 2014). Debido a estas carencias se obstaculiza la posibilidad de adquirir viviendas seguras en áreas permitidas por las instituciones gubernamentales en materia de ordenamiento territorial; además de esto, la especulación del mercado inmobiliario juega un papel determinante en el escaso acceso de suelo y/o viviendas favorables para resguardarse de dichas condiciones adversas. El cambio climático representa un desafío actual para la población, ya que se trata de un fenómeno natural que; con gran injerencia de las actividades humanas, está acelerando e impactando en el proceso de calentamiento y enfriamiento del planeta, acrecentando los problemas socioeconómicos y ambientales actuales. Por ello, es necesario que la sociedad cuente con la capacidad de mitigar y/o recuperarse de los impactos negativos que trae consigo una amenaza climática. 

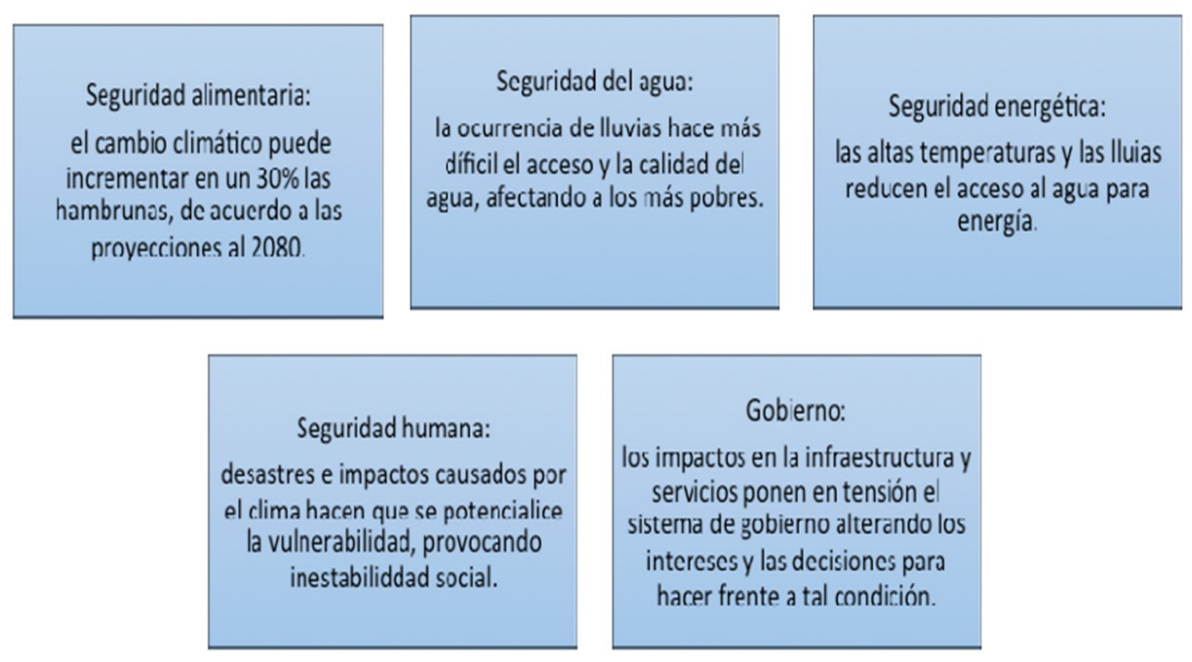

FIGURA 1.

Impactos del cambio climatico y situación de pobreza.

Fuente: Anderson, 2011

Como se aprecia en la figura 1 el cambio climático representa múltiples afectaciones en términos de seguridad de la población, pues las alteraciones que el ser humano ha tenido a mal realizar en el medio natural repercuten en las condiciones climáticas, modificando la estacionalidad y propende a variaciones poco predecibles tanto para las autoridades como para la comunidad directamente afectada, siendo ésta la más dañada. Con recurrencia a lo largo del año; producto de deslaves y desestabilización de taludes en comunidades irregulares, los sectores pobres de la sociedad ven dificultada su condición para hacer frente a este tipo de eventos climáticos; por ende, acrecienta su situación de pobreza.

Cambio climático y pobreza son problemáticas inmanentes entre sí. Los problemas que estos cambios trae consigo son a mediano y largo plazo para las poblaciones pobres: seguridad alimentaria, productividad, viabilidad de los ecosistemas agrícolas a escala mundial, ralentización del crecimiento económico y mayor desigualdad social en zonas urbanas y rurales (Sánchez Vargas, Gay Garcia y Estrada Porrúa, 2011; IPCC, 2014) son algunos de los principales estragos que las diferentes regiones del mundo, principalmente los países en desarrollo, padecen o padecerán de continuar con la dinámica actual de deterioro ambiental. De continuar con esta situación, será necesario contar con mecanismo resilientes ${ }^{[1]}$ de los medios de subsistencia entre la población pobre y marginada, siempre y cuando se cuenten con programas que aborden dicha temática.

\section{POBREZA EN MÉXICO}

Para el caso mexicano el tema de la pobreza es un fenómeno estructural e histórico que ha venido generando adaptaciones a la política nacional. Por ello, el Consejo Nacional para la Evaluación de Política Pública (CONEVAL) considera que la explosión demográfica ha repercutido en gran medida a la existencia de un número mayor de población con algún tipo de pobreza: alimentaria, de capacidades y de patrimonio. Se entiende por pobreza alimentaria aquella población que cuenta con un ingreso per cápita insuficiente para adquirir una alimentación mínimamente aceptable, de acuerdo con los productos contemplados en la canasta básica. El segundo tipo de pobreza se refiere a la población que; si bien puede cubrir sus necesidades mínimas de alimentación, cuenta con un ingreso per cápita insuficiente para realizar las inversiones mínimas en educación y salud de cada uno de los miembros del hogar. Finalmente, aquellos sectores que pueden cubrir sus necesidades básicas de alimentación, educación y salud pero; que cuenta con un ingreso insuficiente para 
adquirir mínimos indispensables de vivienda, vestido, calzado y transporte para cada miembro del hogar, puede clasificarse dentro del tercer tipo de pobreza (CONEVAL, 2013).

De acuerdo con los parámetros de carencia antes mencionados, los estados más pobres del país son Guerrero, Michoacán, Chiapas, Oaxaca y Veracruz. Como resultado de estas condiciones de carencia en México, ha surgido la inquietud de analizar los posibles impactos que tiene el cambio climático sobre la pobreza en el país. En este sentido, los datos de temperatura y precipitación, ligados a la cantidad de población con algún nivel de pobreza, permiten la creación de escenarios poco alentadores: bajas en la productividad agrícola, aumento de costos en bienes básicos de consumo y escasez de alimentos. En otras palabras; ante estas situaciones, se tiende al encarecimiento de la vida, que hará más evidente la brecha entre los que más y los que menos tienen, implicando fuertes procesos de adaptación (de la Fuente y Villarroel, 2013) como los sistemas de enfriamiento y calefacción al interior de la vivienda, así como servicios básicos de electricidad y agua entubada.

En la actualidad, estas condiciones y tipos de pobreza parecen ser cada vez más exigentes para alcanzar mejoría en la condición de vida, sobre todo considerando las tendencias del mercado y el tipo de modelo económico imperante en nuestra sociedad; razón por la cual los países con menor desarrollo, se han preocupado por "combatir" los niveles de pobreza que prevalecen, situación que ha obligado al diseño e implementación de programas sociales que contribuyan a la reducción de la desigualdad social y económica de la población, pero soslayando el factor ambiental como determinante y agente transformador del desarrollo, principalmente cuando las variaciones climáticas se presentan con tal dinamismo.

Sin embargo, esta situación parece tener deficiencias en el manejo y aplicación, pues no se ha logrado reducir los niveles de pobreza (situación que implica exposición a riesgos) debido a las condiciones de vida en la que esta población habita: asentamientos irregulares en zonas de pendiente, materiales poco consolidados en techos y paredes, inaccesibilidad a zonas seguras y nula derechohabiencia al servicio de salud. Según Anderson y Verner (López Feldman, 2013:13) “[...] ]existen otros argumentos para sugerir que los pobres enfrentan las consecuencias más graves del cambio climático que los ricos[...], los pobres tienes más probabilidades de vivir en tierras marginales[...donde] la pobreza y la desigualdad [aumentan] como consecuencia del cambio climático[...]” (ver figura 2).

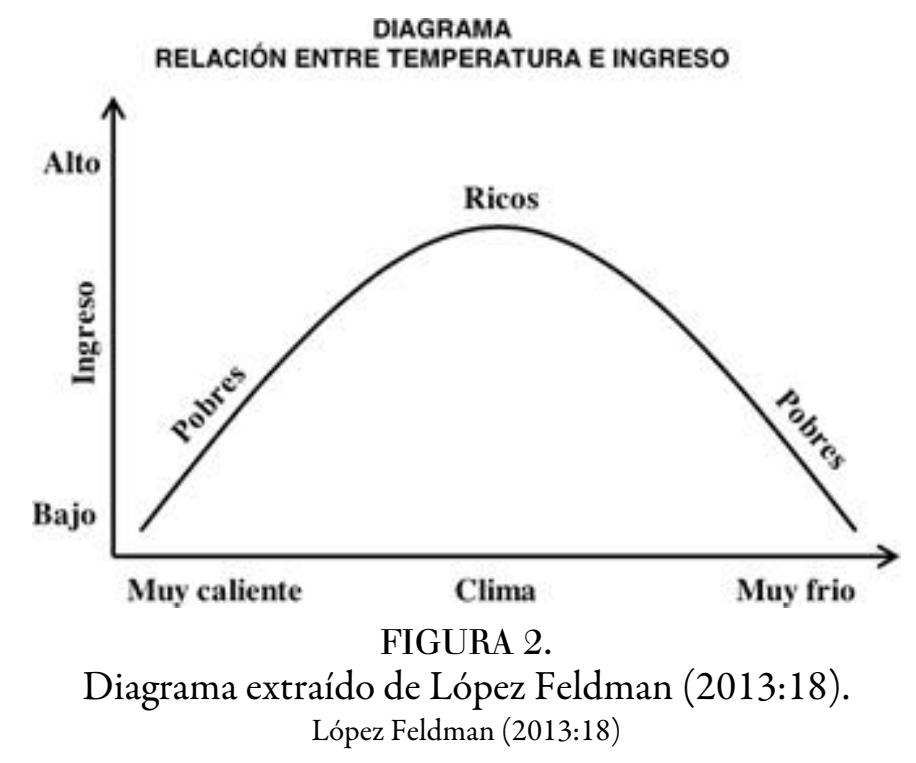

El escenario parece desalentador en cuanto a los niveles de pobreza, situación que trae consigo efectos considerables en cuanto a la agudización de problemas sociales y ambientales que confluyen en el territorio, debido a la heterogeneidad de las características físico-geográficas que por naturaleza le corresponden a México. Si bien existen esfuerzos para el combate a la pobreza por diferentes instancias internacionales 
y nacionales, el discurso ha ido cambiando, pues los efectos del cambio climático arremeten en el empobrecimiento de la población; al no existir mecanismos de defensa y adaptación que hagan frente a este fenómeno sistémico y dinámico, se incrementa la brecha de desigualdad sobre todo en regiones altamente vulnerables a fenómenos naturales como sequías y precipitaciones. Por ello, el desarrollo y crecimiento económico y social se verá mermado en escala mundial, dificultando los procesos de intercambio productivo y disminuyendo la oferta de bienes y servicios a precios accesibles, lo que conlleva a la marginación cada vez más de la población (Tibaldi, 2012).

\section{VULNERABILIDAD FÍSICA Y SOCIAL}

La población pobre depende, en la mayoría de los casos, de bienes y servicios que los ecosistemas proveen tales como alimento, materiales para vivienda y combustible, razón por la cual el grado de degradación ambiental está relacionada con la vulnerabilidad física y social. De acuerdo con Lugo e Inbar (2002), la vulnerabilidad se entiende como aquellos aspectos de la infraestructura humana, donde el espacio vivido es el escenario para combatir el peligro. La vulnerabilidad depende de la preparación y de la capacidad de defensa que tenga la población para hacer frente a una condición adversa natural y/o social.

Winchester (1992) menciona que la vulnerabilidad física de áreas sujetas a fenómenos naturales es un síntoma de su vulnerabilidad económica; al mismo tiempo algunos grupos de personas lo hacen vulnerable a desastres, en cuyo caso solo acentúan las condiciones de vulnerabilidad en términos generales, implicando fuertes procesos para mitigar y contrarrestar tales condiciones. Éstas tienen una base estructural (la pobreza como uno de ellos) y es explicada por medio de los hechos acontecidos a lo largo del tiempo, también puede ser modificado si se consideran las demandas actuales de la población, tomando como contexto predominante la globalización, espacio donde las necesidades de bienes y servicios parecen ser más exigentes y/o limitadas, además que solo satisfacen a grupos minoritarios y a diferentes regiones.

En el campo de las ciencias sociales la vulnerabilidad suele ser un componente que repercute en la condición de pobreza de los habitantes de América Latina. Este concepto es adoptado como reflejo de las situaciones socioeconómicas de la población; por ende, ha sido utilizado con mayor frecuencia en el ámbito intelectual y gubernamental en Latinoamérica; empero, en ocasiones no existe precisión y termina por confundirse con pobreza. Por lo tanto, puede ser explicado mediante dos componentes: el primero, como la inseguridad y condiciones de vida que experimentan las comunidades por el impacto de eventos naturales, climáticos, económicos y sociales y; el segundo, como manejo de recursos y estrategias que son utilizadas para enfrentar los efectos del evento (Pizarro, 2001).

Con la aparición del término vulnerabilidad social, referencia las condiciones de la población ante una amenaza natural, se consideran también los procesos de accesibilidad a los recursos y medios de subsistencia. Una fuente de vulnerabilidad es la marginación dentro de los territorios en desarrollo, haciéndose cada vez más notorios en cuanto a pérdidas materiales y humanas. De acuerdo con el IPCC (en Conde, 2010), la vulnerabilidad se caracteriza por la exposición, sensibilidad y adaptabilidad de cada sistema a los cambios climáticos y a los eventos extremos. Esta exposición del sistema social acarrea serias implicaciones materiales y económicas que muchas veces no son tomadas en cuenta por los gobiernos y los tomadores de decisiones, implicando cambios en la dinámica y reconfiguración territorial.

Para combatir la pobreza, distintas organizaciones internacionales se han dedicado a elaborar instrumentos para la erradicación o reducción de ésta; sin embargo, dichas políticas y estrategias no han considerado la vulnerabilidad física y social de la población expuesta, por lo que poco se podrá realizar en los avances de combate a la pobreza, puesto que las instancias competentes destinan fuertes cantidades económicas para subsanar los efectos de la vulnerabilidad en la población, sobre todo como parte de los impactos del cambio climático en zonas segregadas, limitando los mecanismos de defensa y adaptación (Tibaldi, 2012). 
La vulnerabilidad de las comunidades pobres frente a los impactos del cambio climático no sólo se relaciona con los efectos o desastres directos que se ocasionan, sino que minimizan los servicios básicos como agua potable, electricidad y salud, además de poner en riesgo la seguridad alimentaria, puesto que los rendimientos de productos agrícolas se ven amenazados así como las tierras de cultivo. Fenómenos climáticos y aumento de la vulnerabilidad constituyen las piezas claves para entender y actuar ante los desastres recientemente ocurridos en el mundo (León, Magaña y Guigue, 2010), caracterizados por una heterogeneidad topográfica, cultural y económica, que aumenta y potencializa estos desastres, tanto en comunidades rurales como en la periferia de las zonas urbanas, bajo condiciones de uso de suelo irregular, poco seguros, presentados por cinturones de carencia de diferentes tipos (bienes, servicios, salud, ingresos).

Las condiciones de vida en las comunidades rurales impactan en la producción agrícola, pues las políticas gubernamentales han girado al mercado internacional, ocasionando fragmentación del hábitat por parte de estas comunidades lo que constituye una de las principales causas de vulnerabilidad debido a la pérdida de biodiversidad y cambios de uso del suelo que traen como consecuencia inestabilidad del mismo (Zamora, 2011). Cambios de temperatura y alteración del proceso natural de retención de humedad provoca serios problemas de erosión y pérdida de suelos (OCDE, PNUD, BM, et.al., s/f). Como resultado de esta degradación ambiental, aunado al impacto del cambio climático, se prevé la disminución de alimentos debido a la alteración de los temporales y la nula adaptación de los cultivos ante tales alteraciones, sobre todo en las zonas tropicales y subtropicales como abastecedoras de producción agrícola, mermando los alimentos para autoconsumo y exportación (ver figura 3).

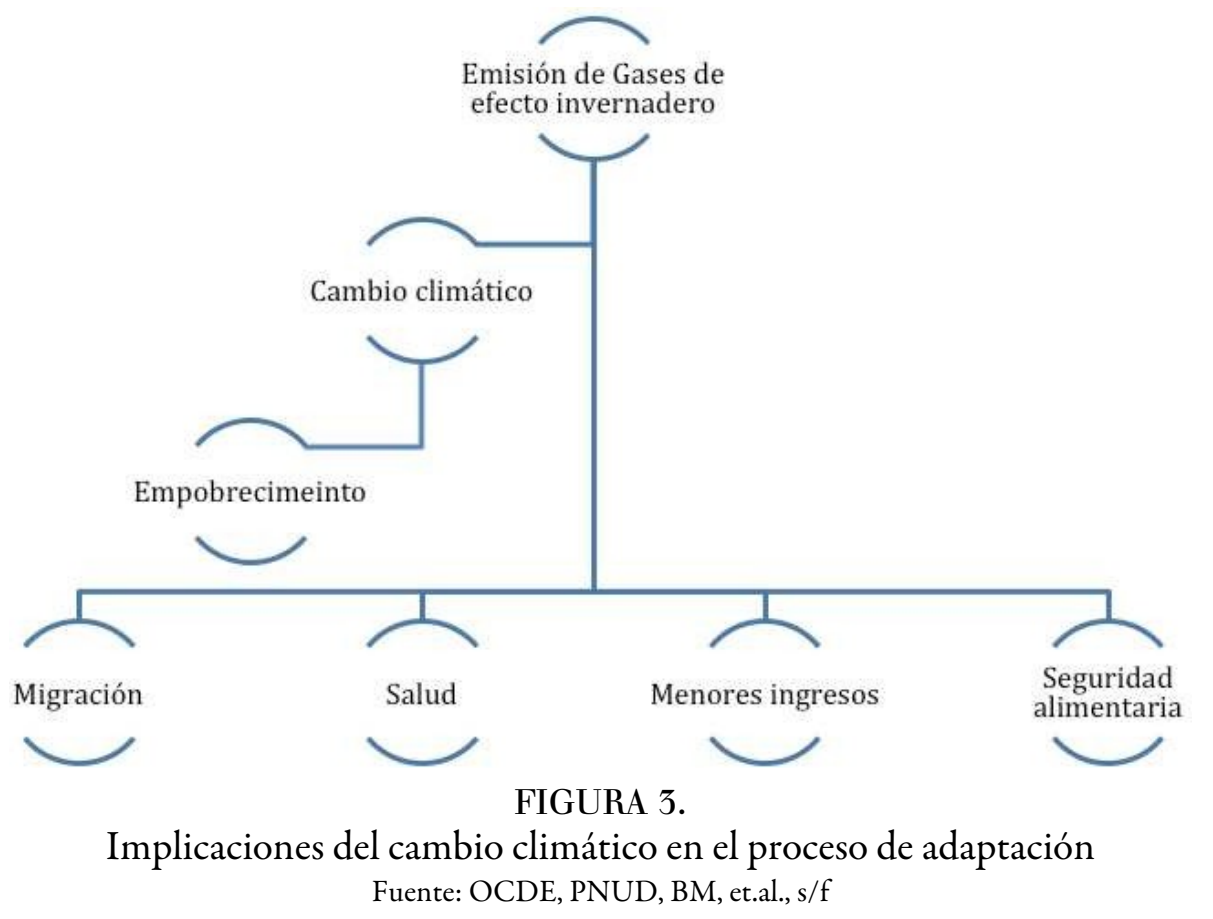

El cambio climático además de representar una seria amenaza a nivel mundial por las consecuencias que se generan de ésta, representa una limitante para el desarrollo, ya que las poblaciones pobres se ubican en zonas geográficamente vulnerables, por lo que sufren los máximos efectos (desastres humanos y materiales), condición que ha quedado invisibilizada por las autoridades y haciendo que cada vez se dificulte la capacidad de respuesta por parte de los afectados y hacedores de política social responsable en materia de prevención de desastres. 


\section{IMPORTANCIA DE LA ADAPTACIÓN COMO MECANISMO DEFENSA}

Un hecho irremediable es que los países realizan acciones para mitigar el cambio climático, mediante la reducción de emisiones de gases de efecto invernadero por un lado, mientras que de manera simultánea se realizan obras de adaptación para las consecuencias del cambio climático; sin embargo, este tipo de infraestructura representa fuertes gastos, inversiones que no toda la población puede llevar a cabo sobre todo considerando que en México, $45.5 \%$ no cuenta con los ingresos mínimos para subsistir; es decir, vive en situación de pobreza (CONEVAL, 2013; BM, 2014).

La adaptación es fundamental en cuanto a cambio climático se refiere. En este sentido, los países en desarrollo deberán enmarcar su política y programas con miras en la reducción de pobreza, sin dejar de lado las estrategias de adaptación de la política económica, social y de protección civil (Anderson, 2011). La condición de adaptación se presenta como imprescindible en regiones pobres, debido a las desigualdades socioeconómicas, culturales y de género, además del limitado acceso a servicios de salud, educación y a las condiciones geográficas características de la ubicación de este tipo de asentamientos humanos carentes de ingresos (CEPAL, 2011), aunado a las condiciones poco favorables que impiden la movilidad o reubicación hacía zonas más seguras, salubres y menos vulnerables en cuestión de riesgo hidrometeorológico.

Desafortunadamente la población pobre es quien se ve afectada constantemente no solo por las consecuencias del cambio climático, sino por los mismos riesgos geológicos y antrópicos que, de cierta manera, se relacionan con esta variación climática de la cual estamos siendo testigos. Estas repercusiones además de poner en riesgo la integridad física de la población, afectan el escaso ingreso y bienes con los que cuentan (Ibíd, 2011). Por lo anterior, es importante la modificación de los programas de desarrollo a escala federal y estatal, pues deben incluir este tipo de amenazas presentes en la dinámica territorial, como agente de cambio social, presupuestal y de configuración espacial. El cambio climático ha venido a representar un gran desafío puesto que se tienen a bien realizar estrategias de ayuda y solidaridad a la población en pobreza para adaptarse a los efectos de estos cambios, mediante un enfoque coordinado e integral, basado en la cooperación internacional (FIDA, $\mathrm{s} / \mathrm{f}$ ) que considere las diferentes repercusiones ambientales y sociales.

Parte de los malos resultados en materia de prevención de riesgos se deben al diseño erróneo de políticas públicas en esta área de intervención gubernamental. Homogeneizar dichas políticas provoca, primeramente, el desface en la implementación de éstas, puesto que al no atender la diversidad natural, cultural y económica de la sociedad en una región determinada, se incrementa la vulnerabilidad ante los embates de los fenómenos naturales, viéndose más dañados aquellos contextos desprovistos, en mayor medida, de los capitales antes mencionados. Sin embargo, sustentar la hechura de políticas públicas (Aguilar Villanueva, 1992) en diagnósticos

superficiales, sin conocimiento preciso del territorio en el que se pretende incidir, deja, tanto en la implementación de las políticas como en las acciones de los programas, tendencias a crear objetivos inalcanzables que generan dos tipos de pérdidas: Pérdidas económicas para el Estado y pérdida de vidas humanas, animales y de flora para la localidad.

La adaptación además de la aplicación de técnicas, requiere un proceso participativo entre los posibles afectados y los tomadores de decisiones, pues la condición social, cultural y económica es muy particular en el caso de la población afectada, pues desafortunadamente, la pobreza repercute en la exposición a este tipo de eventos climáticos (Conde, 2010).

\section{CONCLUSIONES}

El cambio climático impacta directamente en el crecimiento y desarrollo económico y social, pues los desastres ocasionados y producidos por la variación climática, impactan en los costos invertidos para recuperar los daños materiales, por lo que la capacidad de los gobiernos para erradicar la pobreza representa 
un constante desafío (Anderson, 2011). Dicho cambio implica un gran reto, también, para el combate a la pobreza que repercute en la desaparición de los esfuerzos alcanzados en materia de desarrollo social, por lo que es indispensable establecer acciones encaminadas al proceso de adaptación que integre respuestas ante el cambio climático, así como estrategias bajo un desarrollo sostenible.

El diseño e implementación de políticas públicas encaminadas en la atención de los efectos del cambio climático conlleva una acertada hechura de dichas políticas, un conocimiento amplio y minucioso del territorio en el que se pretende incidir e, inmanente a esto, la formación de una sociedad adepta al cuidado del ambiente, así como crear la cultura de la prevención en materia de desastres naturales; por ende, es necesario incorporar acciones y estrategias basadas en el desarrollo sostenible con miras en la evaluación y rediseño de los programas sectoriales actuales, enfocados en la reducción de catástrofes, minimizando la vulnerabilidad de las comunidades ante la exposición de riesgos. La creación de metodologías locales de planificación para hacer frente a los riesgos de la población, sobre todo para reducir la vulnerabilidad de los pobres al mismo tiempo que se otorgan herramientas necesarias para combatir la variabilidad climática, son actividades necesarias para el combate de las consecuencias de estos cambios climáticos abruptos.

El cambio climático no solo conlleva afectaciones al sistema ambiental; más bien, implica altos costos de adaptación en los modos de vida cotidianos con fines de comfort al interior de las viviendas; sin embargo, esta situación es inaccesible para gran parte de la población pobre, generando no solo condiciones poco confortables a los cambios extremos de temperatura y precipitación, sino afectando su seguridad personal y el deterioro de su salud. Empero, la actividad antrópica contribuye en gran medida a la aceleración de este cambio climático.

Resulta imposible adelantarnos a los hechos y a los periodos de estos cambios, sopena de esto, es indispensable modificar la forma de manejarnos ante la situación que aqueja el cambio climático, de tal manera que las decisiones políticas desde la escala global y local impacten de manera positiva en el ambiente y, por ende, en el clima. Las reubicaciones de asentamientos humanos realizados como parte de los programas de gobierno estatal, han sido afectados por las manifestaciones de dicho cambio, pues no se han considerado los factores climáticos, ambientales, geológicos y topográficos en zonas seguras, tampoco los tipos de vivienda adecuadas a las condiciones climáticas de la región, implicando despilfarros del gasto público Anuales por la falta de previsión y legislación en temas ambientales con fines de prevención, mitigación y adaptación.

\section{Bibliografia}

Acuña Gamboa, L. A. (2013). Mirando hacia el cielo (Looking toward sky). ANADIdasko: Revista del Instituto de Estudios de Posgrado, 19, 11-16.

Aguilar Villanueva, L. F. (1992). La hechura de las políticas (1a ed.). México D. F., México: Editorial Miguel Ángel Porrúa.

Anderson, S. (2011). Cambio climático y reducción de la pobreza. Grupo de Cambio Climático en el Instituto Internacional para el Ambiente y Desarrollo. Alianza Clima y Desarrollo. Programa Internacional del Reino Unido.

Banco Mundial. (03 de Marzo de 2014). Banco Mundial. Recuperado el 22 de septiembre de 2014 de http://www.ba ncomundial.org/es/news/feature/2014/03/03/climate-change-affectspoorest-developing-countries

Bauman, Z. (2013). La globalización. Consecuencias humanas (2a ed.). (D. Zadunaisky, Trad.) México D.F.: Fondo de Cultura Económica.

Boltvinik, J., \& Damian, A. (2013). La necesidad de ampliar la mirada para enfrentar la pobreza. Boltvinik, J. \& Damian, A.(Coords): La pobreza en México y el mundo. Realidades y desafíos. México, Siglo XXI editores, $11-42$.

CEPAL (2011). Vulnerabilidad, pobreza y adaptación. La economía del cambio climático en Centroamérica. En Reporte técnico 2011 (págs. 282-316). Chile: CEPAL. 
Conde, C. (2010). El cambio climático observado. En México ante el cambio climático: evidencias, impactos, vulnerabilidad y adaptación (51-57). México: GreenPeace.

CONEVAL. (2013). Consejo Nacional de Evaluación de la Política Social. Recuperado el 17 de junio de 2014 de http ://www.coneval.gob.mx/Medicion/Paginas/glosario.aspx

de la Fuente, A., \& Villarroel, M. O. (2013). The poverty impact of climate change in Mexico. World Bank Policy Research Working Paper, (6461).

FIDA. (s/f). Fondo Internacional de Desarrollo Agrícola. Recuperado el 16 de sepetiembre de 2014 de: www.ifad.org Fromm, E. (2012). Del tener al ser (4a reimp.). México: PAIDÓS.

IPCC. (2014). Cambio climático 2014. Impactos, adaptación y vulnerabilidad. Suiza: PNUD y OMM.

León, C., Magaña, V., \& Guigue, L. (2010). La adaptación al cambio climático:¿ de quién? ȯ para quién?. Siete argumentos para un manual. México ante el cambio climático: evidencias, impactos, vulnerabilidad y adaptación (págs. 57-66). México: Green Peace.

López Feldman, A. (2013). Estudio sobre los impactos del cambio climático en la distribución del ingreso y la pobreza en México. Santiago de Chile: CEPAL, EUROCLIMA y Comisión Europea.

Lugo Hubp, J., \& Inbar, M. (2002). Desastres naturales en América latina (No. 363.34098 D441). Fondo de cultura economica.

Organización de Cooperación y Desarrollo Económico, Programa de las Naciones Unidas para el Desarrollo, Banco Mundial, Programa de las Naciones Unidas para el Medio Ambiente, Banco Africano de Desarrollo, et.al. . (s/ f). Pobreza y cambio climático. Reducir la vulnerabilidad de los pobres mediante la adaptación. Berlín: OCDE, PNUD, BM, PNUEMA, BAD.

Di Virgilio, M. M., Otero, M. P., \& Boniolo, P. (2011). Pobreza y desigualdad en América Latina y el Caribe. Clacso.

Pizarro Hofer, R. (2001). La vulnerabilidad social y sus desafíos: una mirada desde América Latina. Cepal.

Quintero-Ángel, M., Carvajal-Escobar, Y., \& Aldunce, P. (2012). Adaptación a la variabilidad y el cambio climático: intersecciones con la gestión del riesgo. Luna azul, (34), 257-271.

Restrepo, I. (2014). La jornada. Recuperado el 20 de septiembre de 2014 de www.jornada.unam.mx/2014/04/21/ opinion/026alpol

Sánchez Vargas, A., Gay García, C., \& Estrada Porrua, F. (2011). Cambio climático y pobreza en el Distrito Federal. Investigación económica, 70(278), 45-74.

Tibaldi, S. (15 de febrero de 2012). Pobreza y cambio climático tiene un solo camino posible: encontrar un nuevo paradigma . Recuperado el 8 de agosto de 2014 de http://rio20.net/documentos/pobreza-y-cambio-climaticotienen-un-solo-camino-posiblede-salida-encontrar-un-nuevo-paradigma/

Winchester, P. (1992). Power, Choice and Vulnerability. London: James \& James.

Zamora, C. (2011). Crisis rural, cambio climático y pobreza: Hacia la búsqueda de alternativas para la definición de políticas públicas en México. México: Oxfam.

\section{Notas}

1 Para el caso de este trabajo, se entiende por resiliencia aquella "capacidad de los sistemas sociales, económicos y ambientales de afrontar un suceso, tendencia o perturbación peligroso [sic] respondiendo o reorganizándose de modo que mantengan su función esencial, su identidad y su estructura, y conservando al mismo tiempo la capacidad de adaptación, aprendizaje y transformación" (IPCC, 2014:5). 